\title{
ANÁLISE PEDAGÓGICA DE PORTAIS EDUCACIONAIS CONFORME A TEORIA DA APRENDIZAGEM SIGNIFICATIVA *
}

\author{
Sergio da Costa Nunes - Mestre em Ensino de Ciências e Matemática - ULBRA - scnunes@ pop.com.br \\ - Prof. Feevale. \\ Renato Pires dos Santos - Pós-Doutor -Universtaet Karlsruhe, Karlsruhe, Alemanha e Research Institute \\ for Symbolic Computation, Hagenberg, Áustria - renato@ reniza.com - Prof. Adjunto ULBRA.
}

Resumo - As novas tecnologias computacionais, aliadas à disseminação das redes de computadores, em especial a Internet, oferecem diversos meios para auxiliar no processo de aprendizagem significativa, ou seja, podem tornar possível ao aluno construir seu conhecimento através de ações ativas e investigativas. Este artigo estabelece uma relação entre portais educacionais e a teoria da aprendizagem significativa de David Ausubel, procurando fornecer uma base epistemológica para análise pedagógica destes portais. Para tanto, utilizou-se mapas conceituais como ferramenta de análise por atender aos principais pressupostos da teoria de Ausubel, quais sejam os da diferenciação progressiva e da reconciliação integrativa. Espera-se com isto contribuir com um método eficaz para avaliação de portais educacionais.

Palavras-chave: portais educacionais, subsunçores, aprendizagem significativa, mapas conceituais.

\section{PEDAGOGIC ANALYSIS OF EDUCATIONAL PORTALS ACCORDING TO THE THEORY OF THE SIGNIFICANT LEARNING}

\begin{abstract}
The new computational technologies, allied with the dissemination of the computer networks, in special Internet, offer diverse ways to help on the significant learning process and may turn possible to the student to build his own knowledge, through an active and investigative action. This article establishes a relationship between educational portals and the theory of David Ausubel's significant learning, trying to supply an epistemological basis for the pedagogic analysis of these portals. For that, conceptual maps were used as analysis tools for assisting to the main presupposition of Ausubel's theory, namely the progressive differentiation and the reconciliation integrativa. It is our hope that this work will contribute with an effective method for evaluation of educational portals.
\end{abstract}

Key-words: educational websites, subsumers, significant learning, conceptual maps.

\section{INTRODUÇÃO}

O envolvimento significativo do aluno no processo de aprendizagem refere-se ao seu papel ativo, sua motivação para investigar, explorar e compartilhar suas descobertas frente ao grupo, procurando a construção significativa de seus conhecimentos.

$\mathrm{O}$ professor como indutor da aprendizagem significativa, deve ir além da simples transmissão de conteúdos, ele deve traçar estratégias e situações que motivem o aluno na perspectiva de aquisição de conhecimentos relevantes às suas necessidades.

Atualmente, os ambientes informatizados, em especial a Internet, estão sendo utilizados como elementos de apoio à aprendizagem. Na utilização destas ferramentas, o professor deve ser bastante criterioso, pois normalmente as ferramentas pedagógicas

\footnotetext{
*Artigo extraído da dissertação "Sobre a Interação de Portal Educacional e os Conhecimentos Prévios na Aprendizagem de Matemática”, apresentada no PPGECIM-ULBRA em maio de 2006.

V. $4 \mathrm{~N}^{\mathrm{o}} 1$, Julho, 2006
} 
freeware disponíveis em sites e portais voltados ao ensino ainda seguem o paradigma da instrução assistida por computador, disfarçados com a utilização de novas tecnologias de hipermídia (som, imagem, animações etc.).

Neste artigo se apresenta uma investigação sobre a aplicabilidade de portais educacionais na organização de subsunçores, considerando as premissas da abordagem da Teoria de Aprendizagem Significativa de David Ausubel.

\section{APRENDIZAGEM SIGNIFICATIVA}

Para David Ausubel (1980), aprendizagem significativa é o processo no qual uma nova informação é relacionada a um aspecto relevante já existente na estrutura cognitiva do indivíduo. Ausubel (1978) relaciona aprendizagem significativa com significado, estabelecendo uma relação intrínseca entre estes conceitos, relacionando-os com a estrutura cognitiva do aluno, de acordo com os processos inerentes a cada aprendiz.

São necessárias duas condições fundamentais para que ocorra a aprendizagem significativa, a predisposição para aprendizagem significativa e o material de aprendizagem ser potencialmente significativo.

\subsection{Predisposição para aprendizagem significativa}

Para que ocorra a aprendizagem significativa é necessário que o aluno realmente esteja predisposto a estabelecer o relacionamento entre os novos conceitos e os conceitos relevantes disponíveis em sua estrutura cognitiva conforme salienta Ausubel (1978). Caso o aluno não esteja com esta predisposição, mesmo que possua idéias relevantes adequadas aos novos conceitos, estará adquirindo uma aprendizagem mecânica, ou seja, aquela aprendizagem em que os novos conceitos apresentam pouca ou nenhuma relação com os conceitos já existentes em sua estrutura cognitiva.

\subsection{Material de aprendizagem potencialmente significativo}

O material de aprendizagem potencialmente significativo deve possuir duas características básicas de acordo com Ausubel (1978): de natureza substantiva e não arbitrário.

Um material é considerado substantivo quando está relacionado com as idéias pertinentes ao conteúdo desenvolvido, porém, este fator deve estar aliado às idéias relevantes em relação ao tema, já contidas na estrutura cognitiva do aluno, tais idéias servirão de esteio ao novo conteúdo a ser aprendido.

O material não arbitrário é aquele que relaciona-se com a estrutura cognitiva do aluno sem alterar o seu significado. Isto se refere ao fato de determinados símbolos ou palavras apresentarem sempre o mesmo significado para o aluno, independente da ocasião ou formas como estão relacionadas.

\subsection{Os subsunçores e a sua organização}

Pode-se conceituar subsunçor como sendo uma idéia ou conjunto de idéias mais amplas que formam um esteio ou âncora, no qual novas idéias irão se fixar para formar novas proposições mais significativas. Segundo Moreira (1999), a aprendizagem significativa ocorre quando a nova informação ancora-se em conceitos ou proposições relevantes, preexistentes na estrutura cognitiva do aprendiz.

Os subsunçores podem ser organizados de diversas maneiras na estrutura 
cognitiva do indivíduo, verifica-se sua organização especialmente por diferenciação progressiva e reconciliação integrativa.

\section{a) Organização de subsunçores por diferenciação progressiva}

A organização dos subsunçores por diferenciação progressiva, estabelece que o conteúdo deve ser programado de maneira a que as idéias mais gerais e inclusivas venham primeiro, diferenciando-se progressivamente em função dos detalhes e de suas especificidades. Em suma, parte-se dos subsunçores maiores (mais inclusivos), para os subsunçores menores (menos inclusivos).

Parte-se do princípio de que é mais fácil para o aluno aprender as partes de um todo mais amplo do que aprender a partir de partes desconexas, para chegar-se a um conceito mais geral. Também considera-se que as idéias ou subsunçores ocupam a mente do indivíduo de maneira hierárquica, na qual os subsunçores mais inclusivos localizam-se no ápice incluindo os demais conceitos e proposições mais diferenciados.

\section{b) Organização de subsunçores por reconciliação integrativa}

A organização de subsunçores por reconciliação integrativa caracteriza-se pelo fato de que em função de novas informações adquiridas, os subsunçores já existentes, se reorganizem e adquirem novos significados.

Os organizadores prévios ou materiais de ensino podem ser escritos de maneira a facilitar este tipo de organização de subsunçores, para tanto, deve-se salientar de que maneira as novas informações relacionam-se com as informações (subsunçores) aprendidas anteriormente.

\section{PORTAIS EDUCACIONAIS}

Quando se estuda sobre as mais diversas formas de Tecnologias da Informação e Comunicação (TIC) existentes e procura-se relacioná-las com o desenvolvimento intelectual do indivíduo, verificam-se suas implicações pedagógicas. Estas implicações, por sua vez, modificam-se na medida da velocidade com que as TIC's vão surgindo ou aperfeiçoando-se. Na realidade, há o repensar sobre as escolas e universidades dentro desta forma de comunicação. De acordo com Magdalena e Costa (2003), é necessário explorar a pluralidade destes meios com a produção de informações nesse espaço de convivência.

Os Portais Educacionais entram neste contexto tendo que conquistar não apenas os alunos, mas também os professores, que passam a aceitar e aprender a utilizar a Internet em sala de aula e serem, então, incentivadores de seu uso como ferramenta que auxilia na construção do conhecimento. De acordo com Delcin (2005), a prática pedagógica ainda caminha à margem do avanço tecnológico; por isto, muito há o que se fazer no contexto virtual da interatividade, conectividade e hipertextualidade.

Considerando os Portais Educacionais como uma ferramenta pedagógica inserida em todas as etapas do ensino, inclusive no ensino superior, pode-se analisá-la de acordo com a Teoria da Aprendizagem Significativa de David Ausubel. Procura-se situá-los como organizadores prévios do conteúdo desenvolvido ou a ser desenvolvido em sala de aula, sempre que existir a preocupação de uma aprendizagem em que realmente o aluno internalize as informações contidas nos portais, trazendo novos significados à sua estrutura cognitiva.

Quando se desenvolve um portal, a principal finalidade é de atrair e estimular sua utilização por um número cada vez maior de usuários, no caso de um Portal 
Educacional, deve existir a preocupação específica com a motivação do aluno durante o processo de ensino/aprendizagem.

\section{MAPAS CONCEITUAIS}

Os mapas conceituais são organizações gráficas dos conhecimentos, que procuram identificar e relacionar os conceitos existentes na estrutura cognitiva do indivíduo, através das inter-relações destes conceitos formando proposições. De acordo com Ruiz-Primo e Shavelson (1996), a maioria das teorias cognitivas parte da suposição de que a inter-relação de conceitos é uma propriedade essencial do conhecimento.

\subsection{Mapas conceituais hierárquicos}

Os mapas conceituais hierárquicos são baseados na Teoria da Aprendizagem significativa de David Ausubel. Segundo Moreira e Bochweitz (1987), mapas conceituais são diagramas hierárquicos que indicam conceitos e as relações entre esses conceitos.

Estas organizações gráficas podem representar as atividades do currículo de um curso, dos conteúdos de uma disciplina e ainda tornar possível o estudo da interdisciplinar e/ou transdisciplinar de cursos. Com relação ao ensino, podem ser utilizados na avaliação significativa dos conteúdos apresentados, como organizadores de conhecimentos prévios ou subsunçores e também como organizadores prévios das atividades de sala de aula.

Entre as diversas aplicações citadas acima, cabe ressaltar a de Faria (1995), no tocante à avaliação de pesquisas escolares. Utilizando um mapa conceitual padrão elaborado por professores ou especialistas, para posteriores comparações com os mapas elaborados durante as etapas da pesquisa. Conforme Faria,

A teoria da aprendizagem significativa de David Ausubel fornece os princípios teóricos para elaboração dos mapas conceituais. Começando pela seleção dos itens relevantes, na qual Ausubel preconiza que devem ser selecionados os principais conceitos e proposições relevantes a estrutura cognitiva do conteúdo a ser considerado. Proposição é considerada como sendo a interligação de dois ou mais conceitos formando a estrutura de uma sentença com caráter significativo.

Em sua organização, os mapas conceituais utilizam-se do princípio da diferenciação progressiva e do princípio da reconciliação integrativa da Teoria da Aprendizagem Significativa de David Ausubel.

\section{OS PORTAIS EDUCACIONAIS E A APRENDIZAGEM SIGNIFICATIVA}

Para considerar um portal educacional como organizador prévio, deve-se analisá-lo segundo as premissas da Teoria da Aprendizagem Significativa; verificando se ele é um material potencialmente significativo. Para isto verifica-se se o portal é substantivo e não arbitrário. Esta análise pode ser feita com a utilização de mapas conceituais que seguem os pressupostos da diferenciação progressiva e reconciliação integrativa.

Quando o mapa conceitual relacionado ao portal educacional estabelece a reconciliação integrativa, mostra que o portal é substantivo porque possui relacionamentos com as idéias ou conteúdos que serão desenvolvidos. Se o mapa conceitual atende ao princípio da diferenciação progressiva, significa que o portal é não arbitrário, porque seu conteúdo está intimamente relacionado com os conceitos subsunçores existentes na estrutura cognitiva do aluno, ou seja, os conceitos do portal 
estão de acordo com os conceitos a serem aprendidos, sem alteração de seus significados.

De acordo com Nunes(2006), os portais educacionais podem ser investigados a partir de um mapa conceitual padrão do conteúdo a ser estudado, elaborado pelo professor ou especialista no assunto.

Ao analisar se o portal educacional atende aos princípios da diferenciação progressiva e da reconciliação integrativa, verifica-se que o mesmo está de acordo com uma das duas condições para uma aprendizagem significativa: a de que o material é potencialmente significativo. Resta verificar se está de acordo com o segundo pressuposto, ou seja, se o aluno ao navegar neste portal irá sentir-se estimulado para a pesquisa. Neste caso está sendo verificada sua predisposição para a aprendizagem significativa.

Para verificar esta predisposição deve-se avaliar o portal segundo os atributos que estimulam a navegação conforme Dorfmann (2003):

- Conveniência: Facilidade para se obter o que se deseja;

- Confiabilidade: Confiança do usuário em relação ao portal;

- Acessibilidade: Facilidade com que o usuário acessa o portal;

- Atualização: Periodicidade com que os conteúdos são atualizados;

- Variedade de Serviços: Se o portal oferece exatamente o que o usuário necessita;

- Personalização: Se o portal utiliza a linguagem do usuário;

- Interatividade: Possibilidade que o portal oferece de interação entre usuários;

- Navegação: Facilidade do usuário acessar as diversa páginas do portal;

- Conteúdo: Profundidade do assunto desenvolvido pelo portal;

- Design: Impressão gráfica causada ao usuário.

A Figura 1 mostra um exemplo de mapa conceitual padrão, com o conteúdo de Equações Diferenciais, extraído de Nunes (2006): 


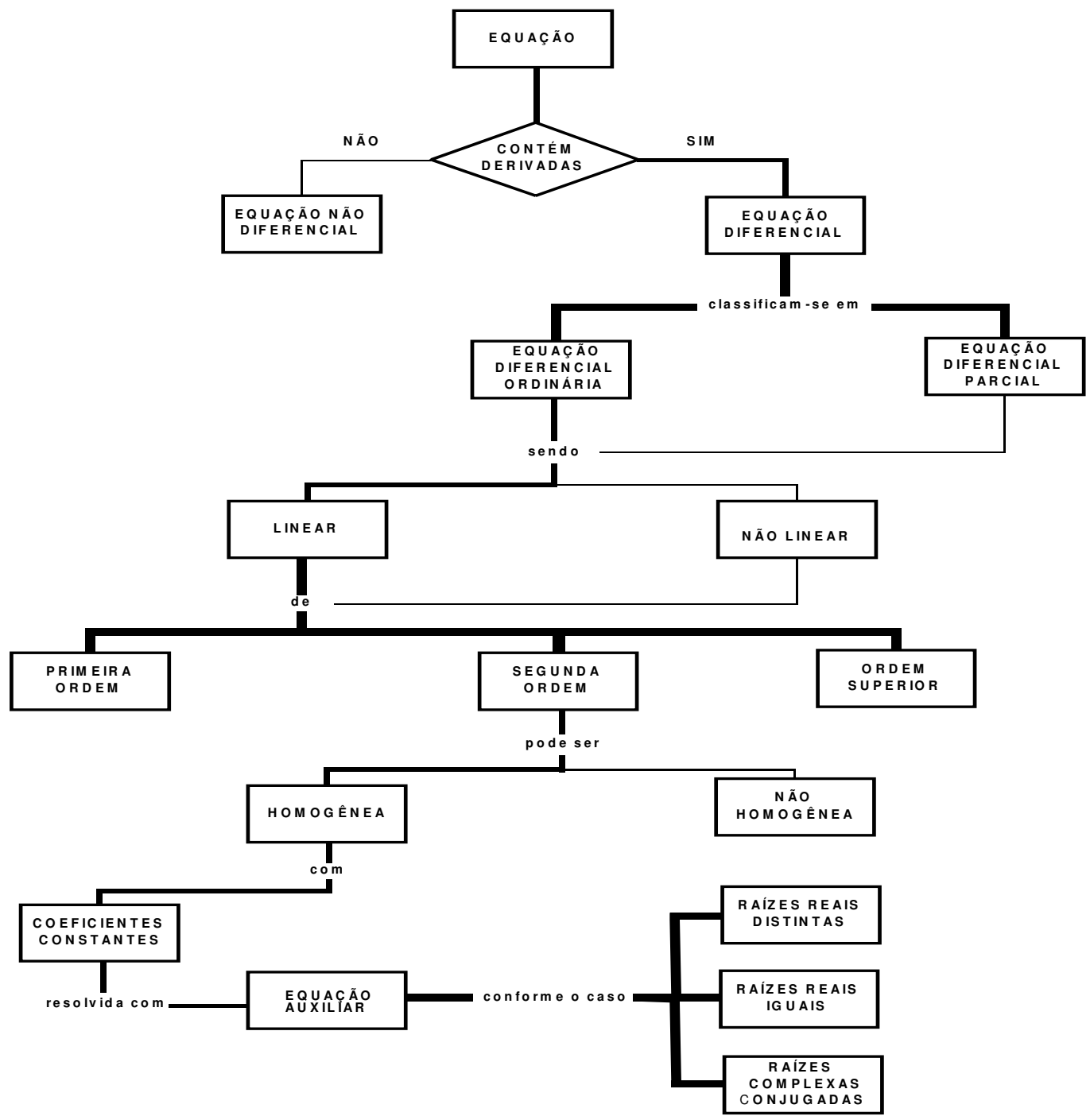

Figura 1- Mapa conceitual padrão

Deve-se considerar que, de acordo com o assunto a ser pesquisado pelo aluno, alguns atributos motivacionais podem adquirir uma maior relevância, esta possibilidade deve ser analisada ao escolher-se o portal educacional para pesquisa.

Para a pesquisa da qual resultou este artigo, foi analisado um portal de matemática com conteúdos das diversas etapas do ensino (fundamental, médio e superior) e, antes da análise pedagógica foi verificado se o portal atendia ao mapa conceitual padrão e continha evidências dos atributos motivacionais.

Como o portal possuía os conteúdos a serem pesquisados pelos alunos de acordo com o mapa conceitual padrão foi feita a análise segundo os atributos motivacionais para navegação:

a) Conveniência e acessibilidade. Na página principal do site existe a relação dos assuntos contidos e também um elemento de busca por palavra-chave, facilitando a navegação.

b) Confiabilidade. Este item pode ser verificado através do contador do número 
de acessos existente na primeira página e também pela variedade de assuntos relativos a área em questão na segunda página.

c) Atualização. A atualização está informada na primeira página, 24 de março de 2005. Por ser um site de ensino de Matemática, as informações não possuem mudanças constantes; pode-se considerar como uma atualização aceitável.

d) Variedade de Serviços. Atende plenamente a este atributo, uma vez que o portal contempla o conteúdo da pesquisa em equações diferenciais.

e) Personalização. A linguagem utilizada no portal está de acordo com os termos matemáticos utilizados, portanto, este atributo está atendido.

f) Interatividade. O portal não contempla este atributo pois não oferece nenhum protocolo de comunicação entre usuários porém, este item não foi relevante para a pesquisa, pois o aluno interagiu diretamente com o portal, na busca dos conteúdos solicitados.

g) Navegação. O portal apresenta-se com um bom sistema de navegação, porém, poderia existir a tecla "voltar" em cada uma das páginas para facilitar a navegação.

h) Conteúdo. O portal atende plenamente a este atributo, contempla todo o conteúdo solicitado para pesquisa.

i) Design. O portal apresenta recursos gráficos que prendem a atenção dos usuários, porém, já existem recursos mais interativos que propiciam movimentos e imagensos.

Selecionou-se então uma turma de alunos do curso superior de Engenharia da disciplina de Matemática III com o conteúdo de Equações Diferencias para a aplicação do teste, seguindo as seguintes etapas:

a) Aplicação de um pré-teste;

b) Elaboração dos mapas conceituais dos alunos com o resultado do pré-teste;

c) Separação em dois grupos homogêneos (controle e experimental)

d) Foi ministrada ao grupo de controle aula expositiva sobre o assunto;

e) O grupo experimental pesquisou no portal selecionado;

e) Aplicação de pós-teste aos dois;

f) Elaboração dos mapas conceituais dos alunos com o resultado do pós-teste;

g) Comparação dos mapas conceituais dos dois grupos com o mapa padrão;

h) Análise qualitativa dos mapas segundo a teoria da aprendizagem significativa.

A seguir, apresenta-se os mapas conceituais do pré e do pós-teste de um aluno do grupo experimental, com a análise feita em função dos subsunçores organizados, também chamados de links cognitivos pelo autor e ainda a análise qualitativa considerando a teoria da aprendizagem significativa de David Ausubel.

\section{Mapas conceituais do aluno quatro do grupo experimental}

a) Mapa conceitual do pré-teste:

No pré-teste aparecem subsunçores organizados com os links 4 e 5 concatenados, o aluno verifica a ordem de uma equação diferencial e define sua homogeneidade com coeficientes constantes e equação auxiliar.

b) Mapa conceitual do pós-teste:

No pós-teste a organização dos subsunçores aumenta com a inclusão do link 3, agora o aluno além de verificar a ordem de uma equação diferencial e definir sua homogeneidade com coeficientes constantes e equação auxiliar, também consegue verificar sua linearidade. 

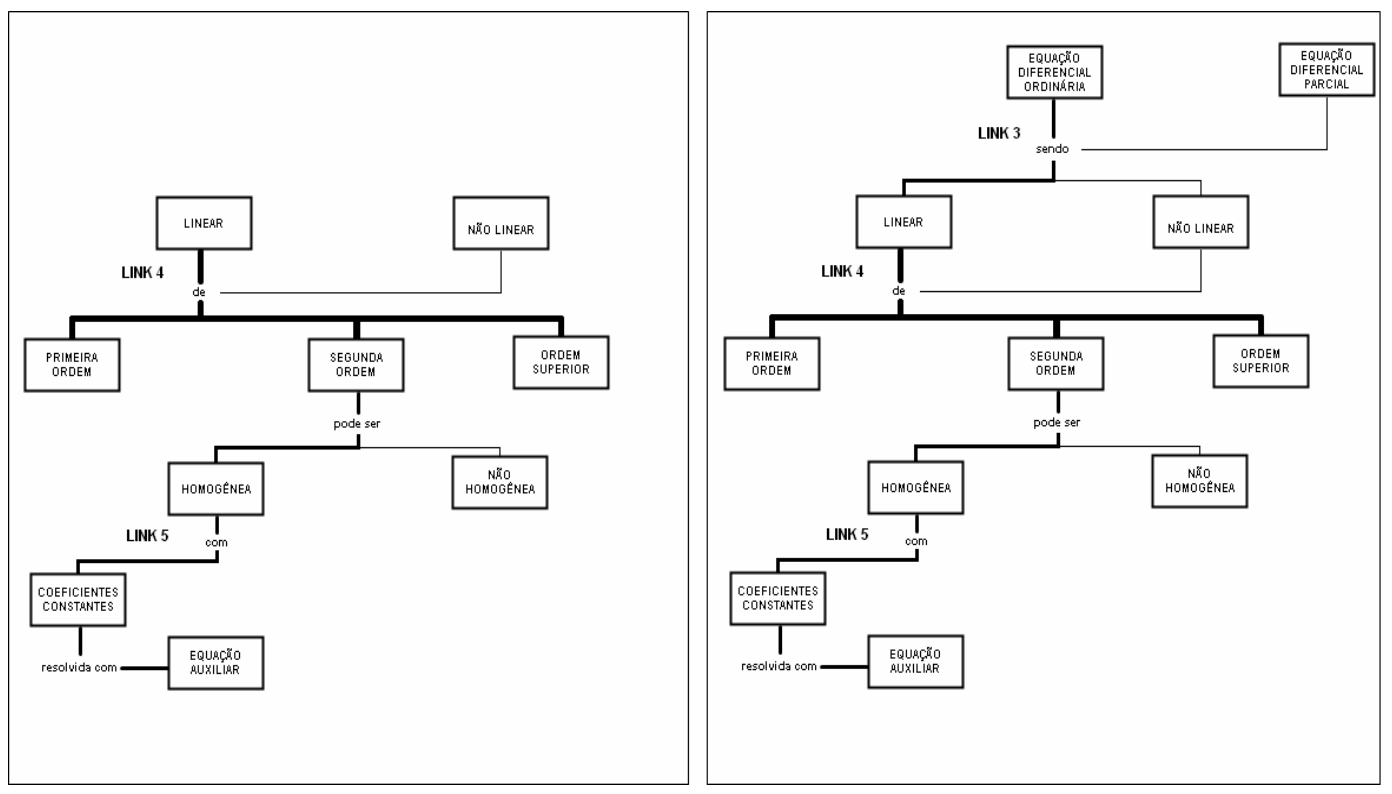

Figura 2 - Mapa Conceitual do Pré-Teste e do Pós -Teste do aluno 4

Em relação ao pré-teste, houve no pós-teste, a organização de mais um subsunçor na estrutura cognitiva do aluno através do link 3, observa-se então que ocorreu aprendizagem significativa não-arbitrária porque o novo subsunçor relacionouse com a estrutura cognitiva, sem alterar seu significado e também que a aprendizagem foi substantiva porque existe uma inter-relação entre os subsunçores. Em função destas duas premissas, pode-se considerar o organizador prévio - portal educacional -, como sendo um material potencialmente significativo para este aluno. Ainda, analisando-se através destas duas premissas de aprendizagem significativa, embora o aluno tenha organizado apenas um subsunçor durante o processo, isto caracteriza a aprendizagem por descoberta, uma vez que o conteúdo não foi formalmente apresentado ao aluno, e sim através do portal.

Como tipo de aprendizagem significativa, considera-se aprendizagem proposicional porque houve nova formação de conceitos com o subsunçor organizado e como forma de aprendizagem significativa considera-se como superordenada, pois o link 3 faz parte do conceito mais amplo de equações diferenciais.

Com a análise qualitativa feita através das comparações entre os mapas conceituais dos dois grupos com o mapa conceitual padrão, pode-se observar uma maior organização de subsunçores no grupo experimental, ou seja, aconteceu uma melhoria na estruturação dos conceitos relativos aos conteúdos pesquisados, na estrutura cognitiva dos alunos do grupo experimental. Com isto, pode-se atestar a efetividade pedagógica do portal educacional em questão.

As tabelas dos subsunçores dos alunos dos grupos de controle e experimental, comprovam que no grupo experimental houve um significativo aumento no total de S.0. - subsunçores Organizados, passando de 27 para 34 enquanto que no grupo de controle não ocorreu aumento.

Tabela 1- Links cognitivos do grupo de controle GRUPO DE CONTROLE 


\begin{tabular}{|c|c|c|c|c|c|c|c|c|c|c|c|c|c|c|}
\hline \multirow[b]{3}{*}{ ALUNO } & \multicolumn{7}{|c|}{ CINTED-UFRGS } & \multicolumn{7}{|c|}{ Novas Tecnologias na Educação } \\
\hline & \multicolumn{7}{|c|}{ PRÉ-TESTE } & \multicolumn{7}{|c|}{ PÓS-TESTE } \\
\hline & Link 1 & Link 2 & Link 3 & Link 4 & Link 5 & Link 6 & SO & Link 1 & Link 2 & Link 3 & Link 4 & Link 5 & Link 6 & so \\
\hline Aluno 1 & & & $\mathrm{x}$ & $\mathrm{x}$ & & & 2 & & $\mathrm{x}$ & $\mathrm{x}$ & $\mathrm{x}$ & & & 3 \\
\hline Aluno 2 & $x$ & & & $x$ & & & 0 & $x$ & & & $x$ & & & 0 \\
\hline Aluno 3 & & & $x$ & $x$ & & & 2 & & & & $x$ & $x$ & & 2 \\
\hline Aluno 4 & $x$ & & $x$ & $x$ & & & 2 & $x$ & & $x$ & $\mathrm{x}$ & $x$ & $X$ & 4 \\
\hline Aluno 5 & $x$ & & $x$ & $x$ & & & 2 & $x$ & $x$ & $x$ & & & $x$ & 3 \\
\hline Aluno 6 & $x$ & & & $x$ & & $x$ & 0 & $x$ & & & $x$ & $x$ & $x$ & 3 \\
\hline Aluno 7 & $x$ & & $x$ & $x$ & & & 2 & & & $x$ & $x$ & & $x$ & 2 \\
\hline Aluno 8 & $x$ & & $x$ & $x$ & $x$ & & 3 & $x$ & & $x$ & $x$ & & $x$ & 2 \\
\hline Aluno 9 & $x$ & & $x$ & $x$ & $x$ & $x$ & 4 & & & $x$ & $x$ & $x$ & & 3 \\
\hline Aluno 10 & $\mathrm{x}$ & $x$ & $x$ & $x$ & $x$ & $x$ & 6 & & & $x$ & $x$ & & & 2 \\
\hline Aluno 11 & $x$ & $x$ & $x$ & $x$ & $x$ & $x$ & 6 & $x$ & $x$ & & $x$ & $x$ & $x$ & 2 e 3 \\
\hline
\end{tabular}

SO - Subsunçores Organizados

Tabela 2 - Links cognitivos do grupo de controle

\begin{tabular}{|c|c|c|c|c|c|c|c|c|c|c|c|c|c|c|}
\hline \multicolumn{15}{|c|}{ GRUPO EXPERIMENTAL } \\
\hline \multirow{2}{*}{ ALUNO } & \multicolumn{7}{|c|}{ PRÉ-TESTE } & \multicolumn{7}{|c|}{ PÓS-TESTE } \\
\hline & Link 1 & Link 2 & Link 3 & Link 4 & Link 5 & Link 6 & SO & Link 1 & Link 2 & Link 3 & Link 4 & Link 5 & Link 6 & so \\
\hline Aluno 1 & $\mathrm{x}$ & & & $\mathrm{x}$ & & & 0 & $\mathrm{x}$ & $\mathrm{X}$ & $\mathrm{x}$ & & & & 3 \\
\hline Aluno 2 & $\mathrm{x}$ & & $x$ & & & & 0 & $\mathrm{x}$ & & $\mathrm{x}$ & & $x$ & $\mathrm{x}$ & 2 \\
\hline Aluno 3 & & & $x$ & $x$ & & & 2 & $\mathrm{x}$ & $\mathrm{X}$ & $\mathrm{x}$ & & & & 3 \\
\hline Aluno 4 & & & & $x$ & $x$ & & 2 & & & $x$ & $x$ & $x$ & & 3 \\
\hline Aluno 5 & & $x$ & $\mathrm{x}$ & $\mathrm{x}$ & & & 3 & $x$ & $\mathrm{X}$ & $\mathrm{x}$ & $\mathrm{x}$ & & & 4 \\
\hline Aluno 6 & & $x$ & $x$ & & $x$ & & 2 & & $x$ & & $x$ & $x$ & $x$ & 3 \\
\hline Aluno 7 & $\mathrm{x}$ & & & $x$ & & $x$ & 0 & $x$ & & & $x$ & $x$ & $x$ & 3 \\
\hline Aluno 8 & & & $x$ & $x$ & $x$ & $x$ & 4 & & & $x$ & $x$ & $x$ & $x$ & 4 \\
\hline Aluno 9 & $\mathrm{x}$ & & $\mathrm{x}$ & $\mathrm{x}$ & $\mathrm{x}$ & & 3 & $\mathrm{x}$ & & $x$ & $\mathrm{x}$ & & & 2 \\
\hline Aluno 10 & $x$ & & $x$ & $x$ & $x$ & $x$ & 4 & $x$ & $X$ & $x$ & $x$ & $x$ & & 5 \\
\hline Aluno 11 & $x$ & $x$ & $x$ & $x$ & $x$ & $x$ & 6 & $x$ & $x$ & & $x$ & & $x$ & 2 \\
\hline
\end{tabular}

$\mathrm{SO}-$ Subsunçores Organizados

\section{CONSIDERAÇÕES FINAIS}

A pesquisa que originou este artigo centrou-se na seguinte situação problemática: Como um portal de ensino pode interagir na organização de conhecimentos prévios ou subsunçores para a aprendizagem em uma disciplina de Matemática do Ensino Superior?

Com base nesta problematização a pesquisa desenvolveu-se de acordo com o objetivo central de investigar a efetividade pedagógica de um portal educacional de Matemática na organização de subsunçores, conforme a Teoria da Aprendizagem Significativa de David Ausubel. Com base neste objetivo geral foram traçados os seguintes objetivos específicos:

a) Selecionar um portal educacional potencialmente significativo e que atenda aos atributos motivacionais, para atuar como organizador prévio.

$\mathrm{Na}$ seleção do portal educacional para utilização na pesquisa, foram verificadas as duas condições para que ocorra a aprendizagem significativa - a predisposição do aluno e a atuação do portal como organizador prévio sendo material potencialmente significativo. As duas condições foram alcançadas.

b) Aplicar mapas conceituais como ferramenta para análise da efetividade do portal educacional.

A escolha de mapas conceituais hierárquicos como ferramenta para avaliar a V. $4 \mathrm{~N}^{\mathrm{o}}$ 1, Julho, 2006 
efetividade do portal educacional deve-se ao fato de que os mesmos são baseados na teoria da aprendizagem significativa que forneceu os princípios teóricos da diferenciação progressiva e reconciliação integrativa para sua organização. Com a elaboração dos mapas dos pré e pós-testes dos alunos dos grupos de controle e experimental, foi possível na comparação com o mapa conceitual padrão, verificar a organização de seus subsunçores e, através da análise qualitativa, verificar a eficácia do portal educacional escolhido na organização dos subsunçores do grupo experimental.

O mais importante é que quando se comprova a efetividade pedagógica de um portal educacional, segundo a teoria da aprendizagem significativa, tem-se a possibilidade de utilizar o portal com mais segurança, porque se estabelece a relação entre o virtual e a organização de novos conhecimentos na estrutura cognitiva do aluno.

\section{REFERÊNCIAS BIBLIOGRÁFICAS}

AUSUBEL, David. Psicologia Educativa: um punto de vista cognoscitivo. México: Trilhas, 1978.

AUSUBEL, David; NOVAK, Joseph; HANESIAN, H. Psicologia Educacional. Rio de Janeiro: Interamericana, 1980.

DELCIN, Rosemeire C.A. A metamorfose da sala de aula para ciberespaço. In: ASSMANN, Hugo. (Org.) Redes Digitais e Metamorfose do Aprender. Petrópolis: Vozes, 2005. p. 56-83.

DORFMANN, Patrícia F. Atributos favoráveis à motivação para visitação de um site: estudo de um portal educacional. 2003. 93 f. Dissertação (Mestrado em Administração) - Escola de Administração, PPGO, UFRGS, Porto Alegre, 2003.

FARIA, Wilson de. Mapas Conceituais: aplicações ao ensino, currículo e avaliação. São Paulo: E.P.U., 1995.

MAGDALENA, Beatriz; COSTA, Íris E. Internet em sala de aula: com a palavra, os professores. Porto Alegre: Armat, 2003.

MOREIRA, Marco Antonio. Teorias de Aprendizagem. São Paulo: EPU, 1999.

MOREIRA, Marco Antônio e BUCHWEITZ, Bernardo. Mapas Conceituais: instrumentos didáticos de avaliação e de análise de currículo. São Paulo: Moraes, 1987.

NUNES, Sergio C. Sobre a Interação de Portal Educacional e os Conhecimentos Prévios na Aprendizagem de Matemática. 2006. 143 f. Dissertação (Mestrado em Ensino de Ciências e Matemática), PPGECIM, ULBRA, Canoas, 2006.

RUIZ-PRIMO Maria; SHAVELSON, Richard. Problems and Issue in the Use of Concept Maps in Science Assessment. Journal of Reseach in Science Teaching. 1996, v. 33, n. 6, pp 569-600. 